\title{
Caso clínico: Tumor Mucinoso Papilar Intra Ductal del Páncreas
}

*Correspondencia:

arturocarpiog@yahoo.com

Teléfono [593] 0986324254

Conflicto de intereses: Los autores declaran no tener conflictos de intereses.

Fondos: Ver la página 72

Recibido: 1 Enero 2018 Aceptado: 11 Diciembre 2018 Publicado: 30 Abril 2019

Membrete bibliográfico: Nieto I, Hernández L, Paladines E, Carpio A. Caso clínico: Tumor Mucinoso Papilar Intra Ductal del Páncreas. Rev. Oncol. Ecu 2019;29(1):67-73.

ISSN: 2661-6653

DOI: https://doi.org/10.33821/256

Copyright Nieto, et al. Este artículo es distribuido bajo los términos de Creative Commons Attribution License, el cual permite el uso y redistribución citando la fuente $y$ al autor original.

\section{Clinical case: Mucinous Papillary Intra Ductal Tumor of the Pancreas}

\author{
Iván Nieto Orellana1, Lucia Hernández Ludueña1, Ernesto Paladines1, \\ Arturo Fernando Carpio Guerrero ${ }^{*}$ *iD
}

1. Servicio de Gastroenterología, SOLCA - Guayaquil.

\section{Resumen}

Introducción: La aparición de nuevas técnicas de imagen y mayor conocimiento de esta entidad han contribuido para que el diagnóstico del Tumor Mucinoso Papilar Intraductal haya aumentado en la última década. En el presente artículo describimos un caso de un paciente con Tumor Mucinoso Papilar Intraductal de páncreas, hallazgo incidental en Tomografía computarizada de abdomen.

Caso Clínico: Se trata de una mujer de 71 años de edad, con antecedentes de Diabetes tipo 2, Hipertensión Arterial Esencial, y cáncer de mama intervenida. En un estudio de control con tomografía computarizada de abdomen se observó un tumor pancreático de $3 \times 1.1 \mathrm{~cm}$. Se realizó una punción transagástrica, con lo que se obtuvo una muestra para estudio patológico, el cual reportó una neoplasia mucinosa papilar intraductal.,

Evolución: La paciente fue sometida a cirugía con la Técnica de Whipple, con duodenopancreatectomía, en donde se retiró la cabeza del páncreas con el tumor adyacente. El estudio de patología confirmó una neoplasia mucinosa papilar intraductal. La paciente no presentó complicaciones post-operatorias y continúa en revisión en consulta externa.

Conclusión: Por tratarse de una lesión maligna, el diagnóstico oportuno y correcto fue importante en este caso, para la conducta terapéutica quirúrgica resolutiva.

Palabras Claves: NEOPLASIAS PANCREÁTICAS, CARCINOMA DUCTAL PANCREÁTICO

DOI: $10.33821 / 256$

\section{Abstract}

Introduction: The appearance of new imaging techniques and greater knowledge of this entity have contributed to the diagnosis of the Mucinous Papular Intraductal Tumor has increased in the last decade. In the present article, we describe a case of a patient with pancreatic papillary intraductal mucosal tumor, incidental finding in abdominal CT scan. 
Clinical Case: This is a 71-year-old woman with a history of type 2 diabetes, essential arterial hypertension, and intervened breast cancer. In a control study with abdominal CT, a pancreatic tumor of $3 \times 1.1 \mathrm{~cm}$ was observed. A transgastric puncture was performed, which obtained a sample for pathological study, which reported an intraductal papillary mucinous neoplasia.

Evolution: The patient underwent surgery with the Whipple Technique, with pancreaticoduodenectomy, in which the head of the pancreas with the adjacent tumor was removed. The pathology study confirmed an intraductal papillary mucinous neoplasm. The patient did not present post-operative complications and continues in external consultation review.

Conclusion: Because it is a malignant lesion, the opportune and correct diagnosis was important in this case, for the operative surgical therapeutic behavior.

Keywords: PANCREATIC NEOPLASMS, PANCREATIC DUCTAL CARCINOMA

DOI: $10.33821 / 256$

\section{Introducción}

Los primeros casos de Tumor mucinoso Papilar intraductal (TMPI) fueron descritos en 1982 por Oashi y Maruyama. Se lo define como una neoplasia epitelial intraductal visible constituida por células productivas de mucina, caracterizada por la dilatación segmentaria o difusa de los ductos pancreáticos, el crecimiento papilar intraductal y la secreción de moco. Las nuevas técnicas de imagen han permitido obtener mejores imágenes tanto del parénquima como de los conductos pancreáticos, lo que ha hecho que esta entidad sea reconocida cada vez con mayor frecuencia [1].

El diagnóstico diferencial es complejo por lo que la pancreatitis crónica obstructiva, cistoadenoma o el adenocarcinoma son las principales entidades que se involucran [2].

Se reporta este caso ya que se trata de un hallazgo casual en un paciente asintomático.

\section{Caso Clínico}

Se trata de una mujer de 71 años de edad, con antecedentes de Diabetes tipo 2, Hipertensión Arterial Esencial, y cáncer de mama intervenida quirúrgicamente y con tratamiento con quimioterapia y radioterapia. En un estudio de control con tomografía computarizada (TC) de abdomen se observó a nivel de cuerpo pancreático una formación hipodensa, lobulada, tabicada y ligeramente heterogénea de $3 \times 1.1 \mathrm{~cm}$.

Este hallazgo en una paciente asintomática motivó la realización de una Ultrasonografíaendoscópica (USE), la cual confirmó la presencia de la lesión, con características heterogénea, solida - quística de patrón interno hipoecoico con varias zonas aneicoicas separada por tabiques, de bordes mal definidos (Figuras 1 y 2), adicionalmente se midió el conducto pancreático el cual tuvo un diámetro de $4 \mathrm{~mm}$. 
Se realizó una punción trans-gástrica con aguja fina número 19 y micropinza, con lo que se obtuvo una muestra para estudio patológico. En el servicio de Anatomía Patológica se reportó que la proliferación papilar consistía en una neoplasia mucinosa papilar intraductal, con signos de leve displasia (Figura 3). Todos los exámenes de laboratorio estuvieron dentro de parámetros normales. La paciente se refirió clínicamente asintomática, y actualmente continúa sin presentar síntomas asociados.

Figura 1. Ultrasonografía-endoscópica



Ultrasonografía-endoscópica, lesión heterogénea, solida - quística de patrón interno hipoecoico con varias zonas aneicoicas separada por tabiques, de bordes mal definidos, que mide $1 \mathrm{~cm}$.

\section{Figura 1. Punción transagástrica}

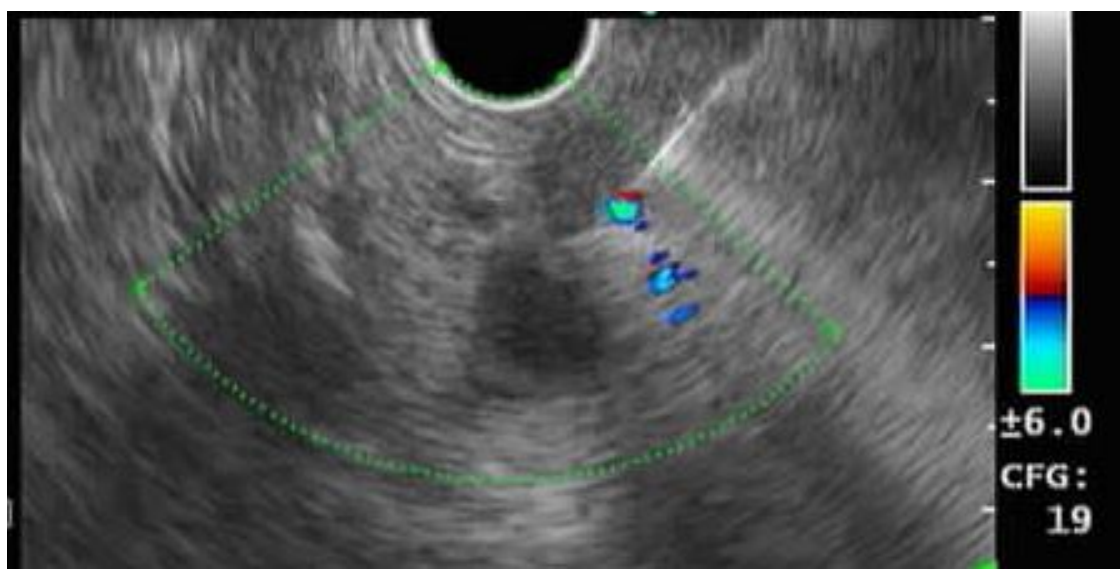

Punción trans-gástrica con aguja número 19 y micropinza, mediante Ultrasonografía-endoscópica. 
Figura 3. Histología

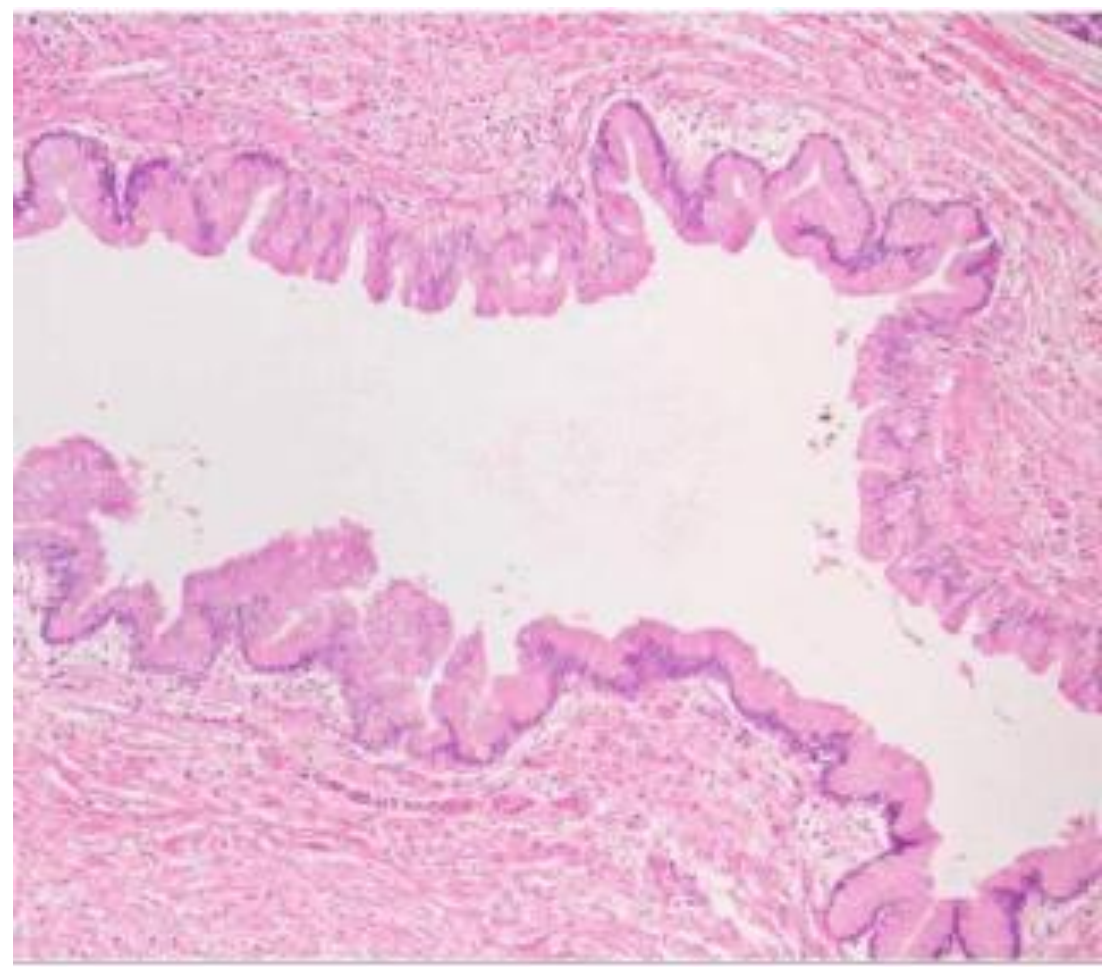

Proliferación papilar consistente con neoplasia mucinosa papilar intraductal, con signos de displasia.

\section{Evolución}

La paciente fue sometida a cirugía con la Técnica de Whipple, con duodenopancreatectomía, en donde se retiró la cabeza del páncreas con el tumor adyacente. El estudio de patología confirmó una neoplasia mucinosa papilar intraductal. La paciente no presentó complicaciones post-operatorias y continúa en revisión en consulta externa.

\section{Discusión}

La prevalencia real de las lesiones quísticas de páncreas sigue siendo una incógnita. Varios estudios han intentado aclarar este punto, resultando en un amplio espectro de prevalencias que va desde $0.2 \%$ - $3 \%$ [4]. Se localizan con más frecuencia en la cabeza del páncreas y alcanzan su máxima prevalencia entre la sexta y séptima década de la vida, tal como en este caso donde nuestro paciente tenía 71 años. Los genes involucrados en la carcinogénesis son el KRAS, p53 y el gen supresor DPC4 [5]. 
En el TMPI el epitelio neoplásico puede ser liso o formar estructuras papilares y puede originarse en el epitelio del conducto pancreático principal, $(21-36 \%)$. Tumor de ramas secundarias, (38 - $48 \%$ ). 0 ambos, (mixto $28-41 \%$ ).

Aproximadamente el $25 \%$ de pacientes con TMPI de conducto principal y los de ramas secundarias, son asintomáticos. El hallazgo es incidental en exámenes de imagen. Cuando son sintomáticos se presentan con dolor abdominal, pérdida de peso, ictericia, pancreatitis, diabetes o esteatorrea. La mayoría de las lesiones quísticas pancreáticas se detectan de forma incidental. La colangiopancreatografía por resonancia magnética [3], es la técnica de imagen de elección para la detección y caracterización de las lesiones quísticas pancreáticas. La USE es otra técnica de elección que presenta una sensibilidad para la detección y caracterización de las lesiones quísticas pancreáticas muy similar a la de la colangio-RM, pero, además, ofrece la oportunidad de una punción aspirativa con aguja fina (PAAF) dirigida por USE ${ }^{7}$, que permite el análisis del líquido intraquístico obtenido. La sensibilidad, la especificidad, el valor predictivo positivo y el valor predictivo negativo de la USE en el diagnóstico de TMPI son del 86, 99, 78 y $99 \%$, respectivamente [9]. Así como se realizó con este paciente que mediante PAAF realizada con la USE se pudo llegar al diagnóstico definitivo.

El análisis citológico de las muestras obtenidas mediante USE-PAAF presenta una precisión diagnóstica solo ligeramente superior a las técnicas de imagen (50-60\%) debido a la baja celularidad que se obtiene habitualmente con la PAAF convencional [5], incrementándose con la obtención de material mediante micropinza (80 \%-90 \%) [7].

Las recomendaciones terapéuticas han cambiado de forma considerable en las dos últimas décadas. La resección se recomienda para todos los casos con TMPI de conducto principal que reúnen la doble condición de ser buenos candidatos quirúrgicos y presentar una buena probabilidad de supervivencia [8]. En los casos de rama secundaria, el manejo en el momento actual es más conservador.

\section{Conclusiones}

El TMPI es una patología más común de lo que se piensa. Se diagnostica muchas veces de forma incidental con algún estudio de imagen, ya que la mayoría de las veces el paciente cursa asintomático. Es de suma importancia tenerlo en mente en el diagnóstico diferencial de las patologías quísticas del páncreas, ya que su carácter pre maligno hace que sea una patología que debe tratarse con rapidez. Su diagnóstico precoz, su correcta evaluación y manejo, puede influir en un pronóstico favorable para los pacientes con dicha entidad.

\section{Agradecimientos}

Un agradecimiento al paciente que autorizó la publicación el presente caso. 


\section{Información adicional}

Nota del Editor

La Revista Oncología Ecu permanece neutral con respecto a los reclamos jurisdiccionales en mapas publicados y afiliaciones institucionales.

\section{Abreviaturas}

USE: Ultrasonografía-endoscópica.

PAAF: una punción aspirativa con aguja fina.

RM: Resonancia Magnética.

TMPI: Tumor mucinoso Papilar intraductal.

\section{Archivos Adicionales}

Ninguno declarado por los autores.

Fondos

Los fondos de la investigación fueron propios de los autores del presente artículo.

\section{Disponibilidad de datos y materiales}

Existe la disponibilidad de datos bajo solicitud al autor de correspondencia. No se reportan otros materiales.

\section{Contribuciones de los autores}

INO, ACG, realizaron la idea de investigación, revisión bibliográfica. LHL, EP recolección de datos, escritura del artículo. INO realizó el análisis crítico del artículo. ACG realizó las correcciones editoriales. Todos los autores leyeron y aprobaron la versión final del artículo.

Aprobación de ética y consentimiento para participar

No aplica a este estudio.

\section{Consentimiento para publicación}

Los autores cuentan con el permiso escrito de la paciente para publicar el caso clínico. 
Información de los autores

Iván Nieto Orellana, Jefe del Servicio de Gastroenterología, Instituto Oncológico Nacional “Dr. Juan Tanca Marengo", Solca-Guayaquil.

Lucia Hernández Ludueña, Médico Tratante del Servicio de Gastroenterología, Instituto Oncológico Nacional "Dr. Juan Tanca Marengo", Solca-Guayaquil.

Ernesto Paladines, Médico Tratante del Servicio de Gastroenterología, Instituto Oncológico Nacional "Dr. Juan Tanca Marengo", Solca-Guayaquil.

Arturo Carpio Guerrero, Médico Posgradista del Servicio de Gastroenterología, Instituto Oncológico Nacional "Dr. Juan Tanca Marengo", Solca-Guayaquil.

https://orcid.org/0000-0001-6150-4438

\section{Referencias}

Abreviaturas en la referencias

DOI: Digital Object Identifier

PMID: PubMed Identifier

SU: Short URL
1. Baltimore Consensus Meeting: A revised classification system and recommendations from the Baltimore consensus meeting for neoplastic precursor lesions in the pancreas. Amer J Surg Path; 2015; 39(12), 1730-1741. DOI: $10.1097 /$ PAS.0000000000000533

2. Tanaka M, Fernandez-del Castillo C, Adsay V, Chari S, Falconi M, Jang J, et al. International Association of Pancreatology: International consensus guidelines 2012 for the management of IPMN and MCN of the pancreas. Pancreatology ; 2012(12):183 -197. DOI :10.1016/j.pan.2012.04.004

3. Scheiman M, Hwang J, Moayyedi P: American Gastroenterological Association technical review on the diagnosis and management of asymptomatic neoplastic pancreatic cysts. AGA Journal; 2015 (148):824848. DOI: 10.1053/i.gastro.2015.01.014

4. Farrell J, Brugge W. Intraductal papillary mucinous tumor of the pancreas. Gastroint Endosc ;2002;(55): 701-714. DOI: $10.1067 / \mathrm{mge} .2002 .123641$

5. Morales V, Kenudson M, Ferrone C, Warshaw A, Lillemoe K, Sahani D, et al. Intraductal papillary mucinous neoplasm of the pancreas in young patients: tumor biology, clinical features, and survival outcomes. $J$ Gastroint Surg; 2018(22) 226-234. DOI: 10.1007/s11605-017-3602-z

6. Lawson R, Hunt G, Giap A, Krinsky M, Slezak J, Tang R, et al. Pancreatic cysts suspected to be branch duct intraductal papillary mucinous neoplasm without concerning features have low risk for development of pancreatic cancer. Ann Gastroenterol; 2015;28(4):487-494. PMID: 26423829

7. Caponi S, Vasile E, Funel N, De Lio N, Campani D, Ginocchi L, et al. Adjuvant chemotherapy seems beneficial for invasive intraductal papillary mucinous neoplasms. Eur J Surg Oncol. 2013 (39), 396-403. DOI: $10.1016 /$ j.ejso.2012.12.005

8. Lim L, Lakhtakia S, Ang T, Vu C, Dy F, Chong V et al. Factors determining diagnostic yield of endoscopic ultrasound guided fine-needle aspiration for pancreatic cystic lesions: A multicentre Asian study. Dig Dis Sci 2013;(58):1751-1757. DOI: $10.1007 /$ s10620-012-2528-2

9. Suzuki R, Thosani N, Annangi S, Guha S, Bhutani M. Diagnostic yield of EUS-FNA-based cytology distinguishing malignant and benign IPMNs: A systematic review and meta-analysis. Pancreatology. 2014 (14), 380-384. DOI: 10.1016/i.pan.2014.07.006 